\title{
Treating specific phobias in young people with autism and severe learning difficulties
}

Article

Accepted Version

Burton, P., Palicka, A. and Williams, T. (2017) Treating specific phobias in young people with autism and severe learning difficulties. The Cognitive Behaviour Therapist, 10. e21. ISSN 1754-470X doi: https://doi.org/10.1017/S1754470X17000241 Available at https://centaur.reading.ac.uk/72788/

It is advisable to refer to the publisher's version if you intend to cite from the work. See Guidance on citing.

To link to this article DOI: http://dx.doi.org/10.1017/S1754470X17000241

Publisher: Cambridge University Press

All outputs in CentAUR are protected by Intellectual Property Rights law, including copyright law. Copyright and IPR is retained by the creators or other copyright holders. Terms and conditions for use of this material are defined in the End User Agreement.

\section{www.reading.ac.uk/centaur}

\section{CentAUR}

Central Archive at the University of Reading

Reading's research outputs online 


\section{Abstract}

Background: Animal phobias in young people with autism spectrum disorders (ASD) and intellectual disability (ID) can provoke behaviours that put them at risk and impede access to the community. Previous studies suggest that CBT including systematic desensitization and modelling are effective for people with ID including those with comorbid ASD. Methods of adapting such treatment to people with little spoken language are not well described, and ethical issues concerning both young people and animals during treatment have not been addressed. Aims: To describe using a case series of consecutive referrals (1) adaptations to systematic desensitization when communication of the young person is impaired and (2) the ethics of involving animals in therapeutic interventions.

Method: Treatment was planned based on the use of systematic desensitization with a hierarchy of feared situations using visual symbols and schedules to communicate with the participants.

Results: Five young people with ASD, severe ID and minimal language were successfully treated for dog phobia. Modifications to treatment included both the use of non-verbal means of communication including printed symbols and graded activities such as jigsaw puzzles and picture matching to present information to clients in a more finely graded format.

Conclusions: The treatment of specific phobias is possible with the use of minimal language provided that a variety of individualized stimuli approximating to the feared objects are developed and the safety of all participants is respected. Further work is required to develop sufficiently flexible treatment protocols, which in turn would enable a randomized controlled trial. 


\section{Learning Objectives}

1. To demonstrate the possibility of treating specific phobias in young people with autism spectrum disorder (ASD) and severe intellectual disabilities (ID) using systematic desensitisation.

2. To draw attention to the use of symbol systems in communication with people with little functional communication.

3. To identify the ethical issues which arise from using live animals in desensitization treatment.

4. To indicate that the length of the intervention may be more variable in the population of those with ASD, severe ID and specific phobias. 
Treating specific phobias in young people with autism and severe intellectual disabilities

Specific phobias are common in people with autism spectrum disorders (ASD). In a study of lifetime prevalence Leyfer et al. (2006) found that about half of children and young people with ASD surveyed by were found to meet criteria for a specific phobia compared with less than $5 \%$ in typically developing children and young people. Phobias in young people with ASD can lead to risky behaviours and impede their access to the wider community (Ricciardi, Luiselli \& Camare, 2006; see also table 1 for specific behaviours of the study participants). Dogs are a common focus of specific animal phobia (Chapman, Fyer, Mannuzza \& Klein, 1993) and reduce access to the community even for people with no other difficulties. Reviews of the evidence on treatment of anxiety disorders in typically developing young people have concluded that cognitive behavioural therapy (CBT) is effective (Reynolds, Wilson, Austin \& Hooper, 2012). Adapted forms of CBT have been extended to populations of young people with ASD and comorbid anxiety disorders (Wood et al., 2009). The methods used include psychoeducation and behavioural components such as systematic desensitization aimed at reducing the anxiety response. Young people with ASD and intellectual disabilities (ID) are typically excluded from trials of CBT because they have social and communication issues that may include difficulties accessing thoughts, emotions and feelings as well as information processing problems. In addition those who have both ASD and ID may have additional communication needs. . Many of the components of CBT (e.g. coping self-talk, verbally mediated coping, affective labelling) rely on verbal concepts before discussion with the therapist (Rosen, Connell \& Kerns, 2016) thus rendering traditional CBT inappropriate for most individuals with ASD and ID.

A number of further issues should be considered when designing an intervention for young people with ASD and ID. First, young people with ASD and ID often have little spoken language and use alternative or augmentative communication (AAC) methods such as the Picture Exchange Communication System. Activities need to be explained by pictures alone and 
presented as a sequence to assist anticipation and learning. However, these methods are often unable to convey complex information (cf. problems with use of CBT referenced in the previous paragraph). Second, the use of long sessions (one hour or more typical of CBT) can be difficult for young people with ASD and ID due to attention problems. Third, it is unclear how far the cognitive components can be addressed. The final consideration in designing an intervention for animal phobia requires that the feared animal takes part in at least some sessions, particularly at the end of treatment. Some people with phobias do not escape ("flight") but will attack the animal instead ("fight") which might endanger the welfare of the animal taking part (Williams, 2009). Therefore, the therapist has to take account not only of the person with the phobia, but also any animal involved. Protection for the animal might involve some form of barrier to prevent the animal being injured.

Potential solutions include the use of the behavioural components of CBT such as "in vivo exposure and participant modelling, with the goal being a graduated, systematic, and prolonged exposure to feared stimuli..." (Zlomke \& Davis, 2008) which have demonstrable efficacy for $\operatorname{dog}$ phobias in adults with ID (e.g. Dansey \& Peshawaria, 2009). Systematic desensitization can be delivered without the use of language because it is designed to extinguish the link between a feared stimulus and the fight/flight response by (1) providing an alternative response (often relaxation), (2) development of a fear hierarchy from least to most frightening situations and (3) work up the hierarchy learning not to respond negatively to the feared stimulus. In the case of the participants in this study we developed the hierarchy based on observations of the their responses to stimuli such as pictures, soft toy dogs, videos of dogs and exposure to dogs at a distance within the school site (see also May, Rudy, Davis \& Matson, 2013). Coping behaviours can also be modeled by other participants in the session to demonstrate effective ways of managing the situation, and the skills taught can be generalised to other dogs and other environments. In planning interventions goals of treatment have to be carefully considered. It may not be appropriate to abolish avoidance completely, so the goal 
might be to recognize potentially dangerous situations (e.g. guard dogs) while being able to encounter dogs in the community. Adapted CBT interventions could therefore be used to treat animal phobias for young people with ID and ASD.

In the school where the authors work, reviews of pupil progress had shown that a number of pupils were unable to access the community due to their phobic reactions to dogs. The psychology department agreed to devise a method for overcoming the phobias, so as to improve the possibilities of community activities. This paper describes a series of cases in which the authors establish a flexible protocol for the treatment of dog phobia in young people with ASD spectrum disorder and intellectual disability and severe communication impairments. The intervention was designed following the recognition that dog phobia was hindering the inclusion of young people with ASD and severe ID in community based activities. The basis of the method is systematic desensitization using a range of stimuli from drawings, to photographs and live animals, together with modelling of coping behaviours.

\section{Methods}

\section{Participants}

Five young people aged between 14 and 19 years with ASD and severe ID (IQ range 2040) participated in the programme which was agreed by the school ethics committee (see Table 1 for characteristics). The young people were resident during the week in a specialist school. Two dogs took part in the treatment sessions (a Jack Russell cross and a Labrador) after training by Pets as Therapy (http://www.petsastherapy.org/). The dogs were not trained to take part in behaviour therapy as such, but were trained to be calm and obedient for work in nursing homes 
and hospices. The training included not startling at rapid movements and learning not to bark unexpectedly.

\section{Intervention}

Most of the pupils at the school are not able to use speech for communication, which dictated that the intervention should be capable of delivery non-verbally Intervention goals were determined jointly with the care and education staff and parents, so that the young person would be able to access the community for activities such as walks in the park and shopping trips in town centres., Our intervention therefore consisted of a hierarchy specific to each individual, reinforced practice and therapist modelling. Treatment sessions lasted no more 30 minutes and took place in an animal husbandry teaching area. Sessions were led by the same therapist (authors AP, PB) and the same animal handler for each young person during each session. In each session the participant initially received a visual schedule which displayed the activities that they would be doing, in the order that they would be doing them. The interventions began with exposure to photographs and pictures of dogs, including jigsaw puzzles. For one participant this phase continued until she was able to manipulate photographs (matching, sorting) without either destroying or hiding them. The others showed no avoidance of pictures. Participants were next introduced to furry toy dogs which they learnt to "groom". The subsequent phase was to expose them to a dog nearby but separated from them by a window, followed by bringing the dog in to the same building. The person handling the dog modelled appropriate behaviours to the situations. In the next phase they were encouraged to take items to the dog handler, groom the dog, walk the dog using a shared lead, walk alongside the dog and walk the dog on their own round a small field. Movement to the next step in the hierarchy only occurred when the young person was able to achieve the previous step without avoidance or obvious fear. In the final stage, the participants were exposed to dogs unexpectedly during their daily tasks. This was regarded as a generalization test, although we did not specifically test behavior outside the premises of the school where the intervention took place. 


\section{Results}

Table 1 shows the fear reactions at the start of the intervention and the goals. At the end of treatment all five participants were able to remain calm when coming across a dog unexpectedly but remained less willing to approach the dogs in a surprise situation than in an expected situation.

The number of sessions taken to treat to criterion as predetermined by the team working with the young person varied between 6 and 25 (see Table 1). Measurement of the outcome was by the therapist working with the young person. In addition the therapy sessions were recorded on video.

\section{Discussion}

The five young people are no longer handicapped by their phobia of dogs. They can access the community without escape behaviour when meeting a dog, as recorded by staff working with them. Anecdotal evidence has shown that the young people have not become over friendly with dogs in the community, and remain calm in the presence of dogs. The sessions were short and progress was enabled by using visual means of communication to explain the activities to the participants. The animals who took part were introduced to the young people gradually. An important component of the intervention was that the dogs had been trained to remain calm, and the interaction with the young people was carefully managed to protect the dogs from kicks and blows which might have been delivered by the young people.

The treatment methods outlined here offer potential for overcoming the phobic responses of people with minimal language and intellectual disabilities. Taken together with Dansey \& Peshawaria (2009) this study provides case series evidence of benefit. For people working with individuals with little language and intellectual disabilities, the use of visual methods of communication alongside systematic densitisation tailored to the individuals' unique reactions to 
feared stimuli offers an effective intervention. It seems likely that the mechanism of action is through learning that coming closer to a feared animal is safe. The use of visual methods of communication may have the unintended consequence of increasing the number and duration of exposure events, hence increasing the apparent effect of the intervention.

The duration of treatment was very variable before the treatment goal was reached. Nevertheless, the total treatment time (up to 11 hours) is similar to many treatment trials of CBT for anxiety in typically developing children and young people (Reynolds, Wilson, Austin \& Hooper, 2012). Further work is required to identify the modifications that may be necessary to write a full protocol and hence enable controlled trials.

Further research to identify the characteristics of people with severe intellectual disabilities and dog phobia would be valuable as a prelude to the design of a randomised controlled trial using a manualised treatment. Such a manual will need to take into account the level of intellectual disability, the level of language skill and the types of phobic responses shown so that an appropriate metric can be developed. The variety of hierarchies used in case studies could be used to devise a behavioural measure of phobic response. For instance, one participant in this study would destroy pictures of feared animals, whereas the others were able to manipulate pictures from the beginning, thus changing the initial stage of the intervention, but also demonstrating that a measure needs to incorporate responses to pictures of feared animals as well as approach to the feared animal. Future studies might also use a wider range of measures of the phobic response, including generalisation to the community and longer follow-ups. Ideally physiological and behavioural response measures would be used as suggested by Davis \& Ollendick (2005). Finally the manual would have to be written in such a way that the intervention continues to include generalisation and maintenance.

\section{Conclusions}

Systematic desensitisation can be combined with augmented communication methods to overcome small animal phobias in young people with comorbid ASD and ID. The sessions 
should be short, but the necessary number of hours of therapy time seems to be similar to that seen in other phobia treatment trials. This study has shown the potential of systematic desensitisation for treating simple phobias in a population for whom there is little evidence based guidance. Further research leading to an adequately powered controlled trial is warranted. 


\section{References}

Dansey, D., \& Peshawaria, R. (2009). Adapting a desensitisation programme to address the dog phobia of an adult on the autism spectrum with a learning disability. Good Autism Practice (GAP), 10(1), 9-14.

Davis, T. E., \& Ollendick, T. H. (2005). Empirically supported treatments for specific phobia in children: Do efficacious treatments address the components of a phobic response?. Clinical Psychology: Science and Practice, 12(2), 144-160.

Leyfer, O.T., Folstein, S.E., Bacalman, S., Davis, N.O., Dinh, E., Morgan, J., Tager-Flusberg, H. and Lainhart, J.E., 2006. Comorbid psychiatric disorders in children with autism: Interview development and rates of disorders. Journal of autism and developmental disorders, 36(7), 849-861.

Mannuzza, S., \& Klein, D. F. (1993). A comparison of treated and untreated simple phobia. Am J Psychiatry, 150, 816-818.

May, A. C., Rudy, B. M., Davis, T. E., \& Matson, J. L. (2013). Evidence-based behavioral treatment of dog phobia with young children two case examples. Behavior Modification, 37(1), 143-160.

Reynolds, S., Wilson, C., Austin, J., \& Hooper, L. (2012). Effects of psychotherapy for anxiety in children and adolescents: A meta-analytic review. Clinical psychology review, 32(4), 251-262.

Ricciardi, J. N., Luiselli, J. K., \& Camare, M. (2006). Shaping approach responses as intervention for specific phobia in a child with autism. Journal of Applied Behavior Analysis, 39(4), 445-448.

Rosen, T. E., Connell, J. E., \& Kerns, C. M. (2016). A Review of Behavioral Interventions for Anxiety-Related Behaviors in Lower-Functioning Individuals with Autism. Behavioral Interventions. 31, 120-143 
Williams, T. I. (2009). Intensive Approaches for Childhood Fears. Clinical Psychology in Practice, 95. In H. Beinart, P. Kennedy \& S. Llewelyn (Eds.), Clinical Psychology in Practice (pp. 95-105). Chichester, UK: Wiley-Blackwell.

Wood, J. J., Drahota, A., Sze, K., Har, K., Chiu, A., \& Langer, D. A. (2009). Cognitive behavioral therapy for anxiety in children with autism spectrum disorders: A randomized, controlled trial. Journal of Child Psychology and Psychiatry, 50(3), 224234.

Zlomke, K., \& Davis, T. E. (2008). One-session treatment of specific phobias: A detailed description and review of treatment efficacy. Behavior Therapy, 39(3), 207-223. 


\section{Recommended Reading}

May, A. C., Rudy, B. M., Davis, T. E., \& Matson, J. L. (2013). Evidence-based behavioral treatment of dog phobia with young children two case examples. Behavior modification, 37(1), 143-160.

McMurtry, C. M., Noel, M., Taddio, A., Antony, M. M., Asmundson, G. J., Riddell, R. P., ... \& Team, H. A. (2015). Interventions for individuals with high levels of needle fear: systematic review of randomized controlled trials and quasi-randomized controlled trials. The Clinical journal of pain, 31, S109-S123.

Ollendick, T. H., Cowart, M. J., \& Milliner, E. L. (2013). Specific phobias. In Pediatric Anxiety Disorders (pp. 113-128). Springer New York.

\section{Supplementary information}

Acknowledgements

We are grateful to the staff, parents and young people who provided time and support toenable this work to proceed.

\section{Ethical statements}

We have abided by the Ethical Principles of Psychologists and Code of Conduct as set out by the APA. Approval was granted by the Senior Leadership of Priors Court Foundation on 17th September 2010. The Senior Leadership of Priors Court School consider ethics applications. Prior to starting the intervention consent is obtained from parents or other appropriate caregivers.

\section{Conflict of interest}

Phillippa Burton, Anna Palicka and Tim Williams have no conflict of interest with respect to this publication.

Financial support 
This research received no specific grant from any funding agency, commercial or not-forprofit sectors. 
Table 1

Table of participant characteristics and number of sessions provided

\begin{tabular}{|c|c|c|c|c|c|c|}
\hline \multirow[t]{2}{*}{ Participant } & \multirow{2}{*}{$\begin{array}{l}\text { Age } \\
\text { (gender) }\end{array}$} & \multirow[t]{2}{*}{ Signs of fear } & \multicolumn{2}{|r|}{ Language } & \multirow{2}{*}{$\begin{array}{l}\text { Number of } \\
\text { sessions }\end{array}$} & \multirow{2}{*}{$\begin{array}{l}\text { Criterion for end } \\
\text { of treatment }\end{array}$} \\
\hline & & & Comprehension & Expression & & \\
\hline Anthony & $16: 5(\mathrm{~m})$ & $\begin{array}{l}\text { Unable to remain in same } \\
\text { space as dog - becomes very } \\
\text { loud and agitated }\end{array}$ & & $\begin{array}{l}\text { Multiple echolalic phrases } \\
\text { from cartoon shows and } \\
\text { nursery rhymes }\end{array}$ & 25 & $\begin{array}{l}\text { Calm behavior in } \\
\text { presence of dog }\end{array}$ \\
\hline Gerda & $15: 5(f)$ & $\begin{array}{l}\text { Very anxious around } \operatorname{dog} s- \\
\text { no longer able to remain on } \\
\text { same side of road }\end{array}$ & & & 19 & $\begin{array}{l}\text { Able to stand } \\
\text { still while dog } \\
\text { passes }\end{array}$ \\
\hline Harriet & $19: 1(f)$ & $\begin{array}{l}\text { Tearing up pictures of dogs; } \\
\text { running away from dogs } \\
\text { even at a distance }\end{array}$ & $\begin{array}{l}\text { Two key word } \\
\text { instructions }\end{array}$ & Three word phrases & 6 & $\begin{array}{l}\text { Able to stand } \\
\text { still while dog } \\
\text { passes }\end{array}$ \\
\hline Oscar & $16: 9(\mathrm{~m})$ & $\begin{array}{l}\text { Unable to leave car when } \\
\text { dog in vicinity }\end{array}$ & Three word level & $\begin{array}{l}\text { Two word level and word } \\
\text { finding difficulties }\end{array}$ & 22 & $\begin{array}{l}\text { Walk past dog } \\
\text { calmly }\end{array}$ \\
\hline
\end{tabular}

